\title{
The construction of linear orderings under conditions of increased memory load
}

\author{
PAUL W. FOOS \\ Florida International University, North Miami, Florida
}

\begin{abstract}
Thirty-nine students constructed linear orderings (e.g., ABCD) from pairwise information (e.g., AB, BC, CD) presented in different orders. Construction took place when only pairwise information was presented, when one additional and irrelevant-to-the-ordering sentence was presented, and when two such additional sentences were presented. The presence of these additional sentences significantly lowered performance, and effects of presentation order were also observed. As predicted, order effects assumed to be due to memory load were differentially influenced by the increased number of presented sentences. Theoretical implications are discussed.
\end{abstract}

Rather than remember individually presented pieces of information, individuals frequently use such information to construct a schema (e.g., Lawson, 1977; Moeser \& Tarrant, 1977). Schema construction is influenced by a number of factors, such as presentation order and sentence type.

The order in which information is presented affects construction. For example, individuals attempting to construct the linear ordering $\mathrm{ABCD}$ from the following three sentences will typically perform differently depending upon the order in which the three sentences are presented.

(1) The A is to the left of the B.

(2) The B is to the left of the C.

(3) The $C$ is to the left of the $D$

Presenting the sentences in the order 1-2-3 (or 2-1-3, or 2-3-1, or 3-2-1) produces relatively good performance. These orders are called match orders because each presented sentence, after the first, contains one new and one old (i.e., previously presented) item. The old item allows the individual to match the information in the new sentence to information in the ordering currently under construction. For example, after learning that $\mathrm{A}$ is left of B (first sentence), one then learns that B is left of $\mathrm{C}$. Thus, the old and matching item, B, allows the individual to add the new item, $\mathrm{C}$, and form a single, integrated $\mathrm{ABC}$ ordering. Match orders always add one item at a time to a single, integrated ordering.

In a nonmatch order (e.g., 1-3-2 or 3-1-2), one presented sentence, after the first, contains two new and, thus, nonmatching items. For example, after learning that $\mathrm{A}$ is left of $\mathrm{B}$ (first sentence), one then learns that $\mathrm{C}$ is left of D. There are no matching items, and one cannot yet construct a single, integrated ordering. Two

Requests for reprints should be sent to Paul W. Foos, Department of Psychology, Florida International University, Bay Vista Campus, North Miami, FL 33181. new items ( $C$ and $D)$ have been added to memory, and they must be kept separate from the previously presented items (A and B). Later, a sentence containing two old items ("The B is left of the C") will allow construction of a single ordering. Nonmatch orders produce lower performance than match orders in large part because of the increase in memory load that occurs when a sentence presents two new items that cannot be immediately added to a single ordering. Reducing memory load by keeping all presented information available to the individual (e.g., on a CRT) reduces the difficulty of nonmatch orders (Foos \& Sabol, 1981).

Differences between the two nonmatch orders (1-3-2 and 3-1-2) are found in some instances but not in others. Such differences appear to result from the loss of separate storage for the nonmatching information presented in the second sentence. When such loss occurs, a difference between the two nonmatch orders is found; when the loss of separate storage does not occur, no difference is found. When information is presented in sentence form, as in the present study, such loss rarely occurs (Foos, 1983).

Finally, differences among the four match orders occur as individuals find it easier to add a new item to the end of an under-construction ordering than to add it to the beginning. Thus, the order 1-2-3, in which new items are always added to the end, is generally easier than the order 3-2-1, in which new items are always added to the beginning (Johnson-Laird, 1972).

This latter effect of presentation order is often offset by the influence of another factor, that of type of sentence (Foos, 1982, 1983). Congruent sentences are those in which the sentence reference point (i.e., "The item is to the left of the reference point") is an old item. These sentences are easier to process than incongruent sentences in which the reference point is a new item. In many studies, including the present one, congruent sentences (easy) are used to add items to the beginning (hard), whereas incongruent sentences (hard) are used to add items to the end (easy). Thus, the effects 
of sentence type and end-beginning offset each other.

Previous work suggests that the difference between match and nonmatch orders is due to memory load and processing. The other differences described above are due primarily to processing. Studies that have reduced memory load (Foos \& Clark, 1983; Foos \& Sabol, 1981) have shown that such reductions reduce matchnonmatch differences but do not significantly alter the other differences assumed to be due to processing. The present study attempted to address this question by increasing, rather than reducing, memory load. Memory load was increased by asking the individual to remember other, additional information while he or she constructed a linear ordering. This increase in memory load should increase the difference between match and nonmatch orders but should not alter any other effects of presentation order.

\section{METHOD}

\section{Subjects}

Thirty-nine introductory psychology students fulfilling a course requirement participated in all conditions of the experiment.

\section{Materials}

Orderings were always composed of the four high-frequency (Thorndike \& Lorge, 1944, AA) nouns "doctor," "farmer," "soldier," and "teacher." All sentences referring to an ordering were of the form "The [noun] is to the left of the [noun]." All six presentation orders for four item orderings were used twice under each of three memory-load conditions. The presentation orders are shown in Table 1. Memory Load 0 (MLO) consisted of the three ordering sentences and no other information. Memory Load 1 (ML1) consisted of the three ordering sentences plus one additional, irrelevant-to-the-ordering, sentence. This extra sentence was presented as the second (half the time) or third (half the time) input sentence. Memory Load 2 (ML2) consisted of the three ordering sentences plus two additional and irrelevant sentences occurring as the second and fourth input sentences. All of these irrelevant sentences were of the form "The [item] lives near a [place]," in which items were the nouns "merchant," "chief," "judge," "king," and "queen," and places were the nouns "hill," "lake," "bank," "town," and "church." All of these nouns are also high-frequency (Thorndike \& Lorge, 1944, AA). The 36 trials (i.e., 6 presentation orders $x$ 3 memory loads $x 2$ occurrences) were randomly ordered, and each trial involved a new, random ordering of nouns.

\section{Procedure}

All participants were run in small groups ranging in size from three to nine. All were told to construct a linear ordering of the words doctor, farmer, soldier, and teacher from the presented sentences and to also remember any other information that might be presented. Each sentence was read to the participants from prepared scripts at a 3-sec rate. Following the last sentence for a given trial, the experimenter said "Recall" and allowed $12 \mathrm{sec}$ for participants to write down the ordering and any other remembered information. A ready signal (i.e., "Okay") was then given, and, $3 \mathrm{sec}$ later, the first sentence of the next trial was presented.

\section{RESULTS AND DISCUSSION}

Table 1 shows mean proportions correct for the three memory-load and six presentation-order condi-
Table 1

Mean Proportion Correct for Memory Load and Presentation Order Conditions

\begin{tabular}{|c|c|c|c|c|c|}
\hline \multicolumn{2}{|c|}{ Presentation Order } & \multicolumn{3}{|c|}{ Memory Load } & \multirow[b]{2}{*}{ Overall } \\
\hline Number & Example & 0 & 1 & 2 & \\
\hline 1 & $\mathrm{AB}, \mathrm{BC}, \mathrm{CD}^{*}$ & .73 & .64 & .53 & .63 \\
\hline 2 & $\mathrm{CD}, \mathrm{BC}, \mathrm{AB}$ & .82 & .69 & .59 & .70 \\
\hline 3 & $\mathrm{BC}, \mathrm{AB}, \mathrm{CD}$ & .76 & .63 & .37 & .59 \\
\hline 4 & $\mathrm{BC}, \mathrm{CD}, \mathrm{AB}$ & .77 & .60 & .51 & .63 \\
\hline 5 & $\mathrm{AB}, \mathrm{CD}, \mathrm{BC}$ & .78 & .51 & .44 & .58 \\
\hline 6 & $\mathrm{CD}, \mathrm{AB}, \mathrm{BC}$ & .73 & .60 & .41 & .58 \\
\hline Overall & & .77 & .61 & .47 & \\
\hline
\end{tabular}

*For purposes of illustration, the correct ordering is always $A B C D$.

tions. A trial was counted as correct only when the constructed ordering and any other presented sentences were correctly recalled.

Over the three memory-load conditions, there was a significant linear trend $[F(1,38)=25.20, p<.01]$. Performance declined as memory load was increased.

There was an overall difference between the four match orders (Orders 1, 2, 3, and 4) and the two nonmatch orders (Orders 5 and 6$)[\mathrm{F}(1,38)=5.21, \mathrm{p}<.05]$. Average performance on the match orders (.64) was better than average performance on the nonmatch orders (.58).

There was no overall difference between add-to-theend (Order 1) and add-to-the-beginning (Order 2) match orders $[F(1,38)=2.38]$. This lack of effect was undoubtedly due to the offsetting effect of sentence type (i.e., congruent vs. incongruent). Likewise, there was no overall difference between the two types of nonmatch orders (Orders 5 and 6$)(F<1)$. Such an effect is rarely found when information is presented in sentence form (Foos, 1983).

Match Orders 1 and 2 produced significantly higher performance than Match Orders 3 and $4[F(1,38)=$ $7.07, \mathrm{p}<.05]$. It appears that participants found it easier to construct the ordering when they could always proceed in the same direction (i.e., always add to the end or always add to the beginning) rather than having to construct in both directions (i.e., add to the end and add to the beginning) during the same trial. This finding has not been reported before and thus may have limited generality. Perhaps it occurs only when individuals construct under conditions of increased memory load. The effect did not, however, interact with the linear trend over memory load $[F(1,38)=1.60]$. Nevertheless, it may be that, when participants expect to receive and/or receive additional information to remember, constructing in both directions is made more difficult.

Finally, there was a significant interaction between the linear trend over memory load and match-nonmatch differences $[F(1,38)=4.96, p<.05]$. As predicted, the difference between match and nonmatch orders increased as memory load increased. This finding supports 
the position that a major difficulty with nonmatch orders is the increase in memory load that they produce. There were no other significant interactions.

In sum, the present results support the hypothesis (Foos \& Sabol, 1981) that differences between match and nonmatch presentation orders are due primarily to memory load, whereas other order effects are due primarily to processing differences. In addition, the results suggest that, under conditions of increased memory load, individuals find it easier to construct in a single direction (beginning or end) and more difficult to construct in two directions (beginning and end).

\section{REFERENCES}

Foos, P. W. (1982). Searching memory for congruent or incongruent information. Journal of Verbal Learning and Verbal Behavior, 21, 108-117.
Foos, P. W. (1983). Using sentences to convey spatial information. Journal of Psycholinguistic Research, 12, 223-234.

Foos, P. W., \& ClARK, M. C. (1983). Learning from text: Effects of input order and expected test. Human Learning: Journal of Practical Research and Applications, 2, 177-185.

Foos, P. W., \& Sabol, M. A. (1981). The role of memory in the construction of linear orderings. Memory \& Cognition, 9, 371-377.

Johnson-LaIrd, P. N. (1972). The three-term series problem. Cognition: International Journal of Cognitive Psychology, 1, 57-82.

Lawson, R. (1977). Representation of individual sentences and holistic ideas. Journal of Experimental Psychology: Human Learning and Memory, 3, 1-9.

Moeser, S. D., \& TARRANT, B. L. (1977). Learning a network of comparisons. Journal of Experimental Psychology: Human Learning and Memory, 3, 643-659.

Thorndike, E. L., \& Lorge, I. (1944). The teacher's wordbook of 30,000 words. New York: Columbia University, Teacher's College, Bureau of Publications.

(Manuscript received for publication May 21, 1984.) 